\title{
Pregnancy-associated cancers: frequency and temporal trends in Italy.
}

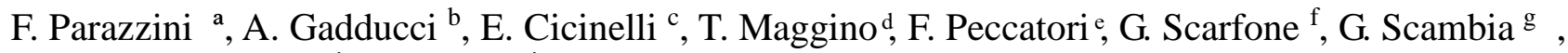 \\ E. Roncella ${ }^{\text {h }}$ P. Zola ${ }^{\text {i }}$, E. Sartori ${ }^{j}$ \\ a Department of Clinical Sciences and Community Health, University of Milan, Italy. \\ ${ }^{\mathrm{b}}$ Department of Clinical and Experimental Medicine, Division of Gynecology and Obstetrics, University of Pisa, Pisa, Italy \\ c 2nd Unit of Obstetrics and Gynecology, Department of Biomedical and Human Oncologic Science, Policlinico University of Bari, Bari, Italy. \\ ${ }^{d}$ Division of Obstetrics and Gynecology, Dell'Angelo Hospital, Mestre, Italy. \\ ${ }^{e}$ Department of Gynecology, European Institute of Oncology (IEO), Milan, Italy. \\ ${ }^{f}$ Gynaecological Oncology Unit, Fondazione IRCCS Ca' Granda Ospedale Maggiore Policlinico, Milan, Italy. \\ ${ }^{\mathrm{g}}$ Department of Gynecologic Pathology, Fondazione Policlinico Universitario A. Gemelli IRCCS, Rome, Italy. \\ h Department of Gynaecology Unit, Fondazione Istituto di Ricovero e Cura a Carattere Scientifico (IRCCS) Ca' Granda Ospedale Maggiore \\ Policlinico, Milan, Italy; Department of Clinical Sciences and Community Health, Università degli studi di Milano, Milan, Italy. \\ ${ }^{\mathrm{j}}$ Department of Obstetrics and Gynecology, University of Brescia, Brescia, Italy.
}

\begin{abstract}
Objective

Very few data are available from large series on the frequency and the time trends of pregnancy associated cancers (PAC), in particular few data are available from Southern European countries, an area characterized by different cancer incidence that north European and American ones. In this paper we have analyzed the frequency and the time trends of PAC in Italy.
\end{abstract}

Methods

This is a population based linkage study using the regional hospital discharge forms (HDF) database of four Italian regions (Piemonte and Veneto (Northern Italy), Tuscany (Central Italy) and Puglia (Southern Italy), regions with more than 17,000,000 inhabitants.

All resident women with a HDF reporting a birth or abortion in the considered period were identified. Risk of developing a pregnancy related cancer was calculated as the ratio of the number of pregnancy related cancers to the total number of pregnancies.

During the period 2003-15 for Piemonte and Puglia, 2006-2015 for Tuscany and 2005-2015 for Veneto a total of 2297648 pregnancies were identified. Overall, the PAC frequency was 134,1 per 100,000 pregnancies: the value ranged from 127,1 in Puglia to 157,3 in Tuscany. The frequency for 100,000 pregnancies was 66,4 in women aged less than 30 years and increased with age being 275,6 among women aged 40 years or more. Approximately two thirds of cancers were associated with pregnancies resulting in a delivery and one third with pregnancies resulting in an abortion. No clear trend emerged in the risk of PAC per 100,000 pregnancies and calendar year.

\section{Conclusion}

This large population-based study offers sound estimates of the frequency of PAC in Italy and suggest that no time trend occurred in the frequency of PAC in Italy during the last ten years. 


\section{Introduction}

Pregnancy-associated cancer (PAC) is generally defined as a diagnosis of cancer made during pregnancy. Even in cancers diagnosed in the year after the end of the pregnancy, the tumor could have originated before or during the pregnancy. Moreover, the pregnancy may have influenced the timing of the diagnosis, thus diagnosis within 12 months of delivery/abortion is considered among PAC.

In the recent years great attention has being given to PAC. The diagnosis of a neoplastic disease in pregnancy, though rare, is a condition of great human and social relevance and of complex management. In many cases the diagnosis of cancer during pregnancy is delayed: signs and symptoms may overlap and be masked by physiological changes that occur during pregnancy [1].

Moreover, systemic medications for cancers have many potential risks and complications for the mother and the baby. A recent cohort study shows that babies exposed to antenatal chemotherapy might be more likely to develop complications specifically for gestational age and admission in neonatal intensive care unit [2].

Although an incidence of about 1 case out of 1000 pregnancies is generally reported [3], few epidemiological studies have estimated the true occurrence of PAC [4,5].

The crude incidence rates were 1, $4 / 1000$ maternities in Australia [4], and 0.9/1000 births in California [5]. Recently, the PAC incidence in Denmark has been set at 0.89/1000 pregnancies, with an estimated annual $1.6 \%$ increase[6].

Scanty information is available on the frequency of PAC in Southern European countries. Some of us have recently published an analysis from Lombardy, a Northern Italian region [7]. This analysis, although based on a population of about ten millions inhabitants, included only 1475 PAC cases. Further, no data have been published from Central or Southern Italy, areas characterized by different reproductive patterns and incidence of neoplasia.

In order to offer more sound estimates of PAC frequency and data from different Italian areas representative of all the three main geographic areas (North Centre and South) characterized by different lifestyle habits and incidence of cancer [8], we have evaluated the incidence and determinants of PAC by cancer site in Veneto, Piemonte, Tuscany and Puglia, four Italian regions with overall more than $17,000,000$ inhabitants. 


\section{Methods}

The present analysis included information from four Italian regions (Piemonte and Veneto (Northern Italy), Toscana (Central Italy) and Puglia (Southern Italy). The participating regions have overall more than $17,000,000$ inhabitants.

To identify all pregnancies and PAC, we used the regional hospital discharge forms [Scheda di Dimissione Ospedaliera (SDO)] database, which stores hospital discharge records from all hospitals placed in the participating regions. These forms contain information about patients and their hospitalization (both ordinary and in day hospital), including, among others, the unique anonymous patient's identification code, demographic characteristics, admission and discharge dates, the main diagnosis and 5 secondary diagnoses [coded according to the International Classification of Disease, Ninth Revision (ICD-9)], date and type of up to 5 interventions, and hospitalization-related costs [coded according to the national diagnosis-related group (DRG) system] [9].

Pregnancies were identified by selecting all SDOs reporting DRG codes related to both deliveries (DRG codes: 370-375) and abortions (DRG codes: 380-381). We further verified that each selected SDO reported diagnoses or interventions related to delivery or abortion. We defined the discharge date of hospitalization as the date of pregnancy outcome (delivery or abortion). Pregnancy associated cancer was defined by any diagnosis of malignant cancer during pregnancy; if it occurred within 9 and 3 months before the date of delivery or abortion, respectively, or if it occurred within 12 months after the pregnancy outcome. Cancers were identified by selecting all SDOs reporting a diagnosis of malignant cancer (ICD-9 codes:140.-208.) in the main or secondary diagnoses. To select only incident cases, we did not consider SDOs in which the cancer was recorded as secondary diagnosis or if a previous SDO reported cancer as the main diagnosis.

For each woman, we selected the first SDO reporting a cancer diagnosis during the observation period, and we defined the admission date as the date of cancer diagnosis. We classified the cancer site on the basis of ICD-9 codes, combining them into the following anatomic groups: head and neck (ICD-9 140.-149., 160.), stomach (ICD-9 151.), colorectum (ICD-9 153.-154.), pancreas (ICD-9 157.), other gastrointestinal tract (ICD-9 150., 152., 156., 158.-159.), lung (ICD-9 162.), other respiratory tract (ICD-9 161., 163., 165.), skeletal/connective tissue (ICD-9 170.-171.), melanoma (ICD-9 172.), skin (excluding melanoma) (ICD-9 173.), breast (ICD-9 174.), uterus (ICD-9 179.), cervix (ICD-9 180.), placenta (ICD-9 181.), endometrium (ICD-9 182.), ovary (ICD- 
9 183.), other gynecologic tract (ICD-9 184.), urinary tract (ICD-9 188., 189.1-189.4, 189.8-189.9), kidney (ICD-9 189.0), nervous system (ICD-9 191.-192.), thyroid (ICD-9 193.), lymphoma (ICD-9 200.-202.), multiple myeloma (ICD-9 203.), leukemia (ICD-9 204.-208.), and other or ill-defined (ICD-9 164., 190., 194.-199.).

We categorized cancers with respect to the period of diagnosis: during pregnancy, if the date of cancer diagnosis preceded the date of pregnancy outcome, and after pregnancy, otherwise.

We computed the ratio of the number of pregnancy-related cancers to the total number of pregnancies $[4,5]$.

The incidence rate of pregnancy-related cancer will be computed as the ratio of the number of pregnancy-related cancers to the total years of follow-up (15 months for an abortion, 21 months for a delivery).

If a pregnancy-associated cancer occurred, follow-up was stopped at the date of cancer diagnosis. We stratified the incidence rate of pregnancy-related cancers by calendar year, age at pregnancy, pregnancy outcome, and nationality.

\section{Results}

During the period 2003-2015 (2003-15 for Piemonte and Puglia 2006-2015 for Tuscany and 20052015 for Veneto) a total of 2.297.648 pregnancies were identified from the SDO database that referred to women aged between 12 and 55 years and residing in the considered Regions.

Among these pregnancies, 1.132.283 ended with a delivery and 795.769 with an abortion.

After exclusion of women with: -a diagnosis of cancer as a secondary diagnosis, -a primary cancer diagnosis before the pregnancy, and women for which the cancer site was unclear, we identified 3058 women with a pregnancy related cancer diagnosis.

Table 1 shows the number of cases and the frequency per 100,000 pregnancies in the total series and according to Region and selected factors.

Overall, the risk was 134,8 per 100,000 pregnancies: the value ranged from 127,1 in Puglia to 157,3 in Tuscany.

Slightly less than $50 \%$ of women diagnosed with cancer were aged less than 35 years. The risk for 100,000 pregnancies was 66,4 in women aged less than 30 years and increased with age being 275,6 among women aged 40 years or more.

Approximately two thirds of cancers were associated with pregnancies resulting in a delivery and one third with pregnancies resulting in an abortion. Italian women had an higher risk as compared with foreign women (respectively, 142,6 versus 90,6 per 100,000 pregnancies). 
Finally no clear trend emerged in the risk of PAC per 100,000 pregnancies and calendar year.

The most common cancers were breast cancer (827 cases, 40.9 per 100,000 pregnancies), thyroid cancer (526 cases, 22,9 per 100,000 pregnancies), and lymphomas (260 cases, 11.3 per 100,000 pregnancies). Skin cancer accounted for 449 cases (19.5 per 100,000 pregnancies), including 227 cases of melanoma and 222 cases of other skin cancers.

Cervical cancer was the fifth diagnosis in frequency (113 cases, 4,9 per 100,000 pregnancies )

Table 1. Number of pregnancies, pregnancy-associated cancers and risk of pregnancy-associated cancer according to selected factor.

\begin{tabular}{|c|c|c|c|c|}
\hline & $\begin{array}{l}\text { Number of } \\
\text { pregnancies }\end{array}$ & $\begin{array}{l}\text { Number of } \\
\text { pregnancy } \\
\text { associated } \\
\text { cancers }\end{array}$ & $\begin{array}{l}\text { Risk of pregnancy- } \\
\text { associated cancer } \\
\text { per } 100,000 \\
\text { pregnancies. }\end{array}$ & $\begin{array}{l}95 \% \text { confidence } \\
\text { intervals of risk }\end{array}$ \\
\hline Total & 2297648 & 3097 & 134,8 & $3111-3147$ \\
\hline \multicolumn{5}{|l|}{ Regions } \\
\hline Piemonte & 617309 & 812 & 131,5 & $858,4-877,6$ \\
\hline Puglia & 683273 & 867 & 127,1 & $962,1-981,9$ \\
\hline Tuscany & 368089 & 579 & 157,3 & $651-668,4$ \\
\hline Veneto & 630066 & 839 & 133,2 & $858,4-877,6$ \\
\hline \multicolumn{5}{|l|}{ Maternal Age $(y r)^{\circ \circ}$} \\
\hline$<30$ & 687231 & 456 & 66,4 & $494,5-509,8$ \\
\hline $30-34$ & 626223 & 787 & 125,7 & $858,4-877,6$ \\
\hline $35-39$ & 462101 & 857 & 185,5 & $962,1-981,9$ \\
\hline $40+$ & 151683 & 418 & 275,6 & $442,4-456,8$ \\
\hline \multicolumn{5}{|l|}{ Nationality $^{\circ \circ}$} \\
\hline Foreign & 387472 & 351 & 90,6 & $389,9-403,2$ \\
\hline Italian & 1476920 & 2106 & 142,6 & $2090-2120$ \\
\hline \multicolumn{5}{|l|}{ Pregnancy outcome $e^{\circ \circ}$} \\
\hline Abortion/miscarriage & 795769 & 993 & 124,8 & $962,1-981,9$ \\
\hline Delivery & 1132283 & 1525 & 134,7 & \\
\hline \multicolumn{5}{|l|}{ Calendar year } \\
\hline $2003^{\circ}$ & 103987 & 117 & 112,5 & $123-131$ \\
\hline 2005 & 162319 & 189 & 116,4 & $230,8-241,8$ \\
\hline 2010 & 164001 & 203 & 123,8 & $230,8-241,8$ \\
\hline 2015 & 129878 & 146 & 112,4 & $166,5-175,9$ \\
\hline
\end{tabular}

${ }^{\circ}$ including data from Piemonte and Puglia only; ${ }^{\circ}$ including data from Piemonte Puglia and Veneto only

\section{Discussion}

To our knowledge only two studies in Australia and California and a study in Lombardy [4,5,7] have systematically analyzed PACs in a large-scale population. For PACs, as for non-PACs, the frequency by site differs in different areas of the world. This paper, that is among the largest 
published analysis on this issue including about 3,000,000 maternities, offers further data from a population of a Southern European country. In particular, it offers data from different Italian regions including regions of Centre and South of the country. Moreover, it covers a more recent period than data from Australia or California.

Before briefly discussing our results, limitations and strengths should be considered.

The timing of diagnosis of cancer is defined by the first hospital admission, which may introduce some imprecision in the timing. We are confident that our data are representative of all the pregnancy related cancers occurring in the considered regions among residents. In fact, all hospital admissions and surgical procedures are registered by law in a regional administrative database. All medical records were reviewed by local medical officers for administrative reasons, and thus, diagnostic accuracy should be warranted.

Among the strengths of this analysis we have to consider the population-based design and the opportunity to analyze data collected for 10 years using similar methods in a population of more than $17,000,000$ of inhabitants.

The present results confirm previous estimates of incidence of PAC.

In a large population-based linkage study conducted between 1994 and 2008 in Australia including more than 1,300,000 maternities, the crude incidence rate of pregnancy-associated cancer was 1.4 per 1000 maternities. In a study conducted in California during the period 1981-1999, the occurrence rate of invasive malignancies was 0.9 of 1000 births.

In the previously quoted Italian study conducted in Lombardy, a region in Northern Italy close to Piemonte and Veneto during the period 2001-2012, the risk of pregnancy-related cancer was 1.2 per 1000 pregnancies.

Analysis from the Australian population [4] has suggested that the crude incidence rate of pregnancy-associated cancer is increasing. We did not confirm this finding. In our study, the frequency of pregnancy-related cancer did not increase with calendar period despite the increasing maternal age at pregnancies observed in the considered period in Italy .

The incidence of various types of cancers among women of reproductive age varies worldwide.

Along this line, it is of interest to analyze the association between cancer and pregnancy in different populations, in particular in Southern European populations, which have been rarely considered in the published epidemiological studies.

Breast cancer is generally reported to be the most common pregnancy related cancer in all the populations. It accounts for about one third of all pregnancy-related cancers. We confirm these findings. In an Australian study and in a Swedish population-based study, melanoma was the most common cancer in pregnancy [4]. Interestingly, in our population melanoma was only the fourth 
diagnosis in frequency. Part of these differences may be due to the implementation of early diagnosis/screening programs for melanoma in Australia and to the different incidence of this disease in different populations. Along this line, cervical cancer is only the fifth diagnosis despite the fact that in developing countries, but also in Sweden, cervical cancer has been reported among the most common malignant neoplasm during pregnancy [10].

In our study, the distribution of diagnoses of PAC was not dissimilar with that reported in nonpregnant patients of similar age [8] thus suggesting that pregnancy does not increase the incidence of any specific tumor.

In conclusion, this large population-based study shows that the frequency of PAC is similar in different Regions of Italy without significant discrepancies with epidemiological data collected in Australia and Sweden. The distribution of diagnoses reflects the incidence of cancer in reproductive age in Italy. Finally no clear time trend in the frequency of PAC was observed in Italy during the last ten years.

\section{Conflict of interest statement}

The author has no conflict of interest to disclosure.

\section{Author contribution section}

All authors contributed to the design and safe conduction of this clinical trial.

\section{References}

[1] J. de Haan, V. Vandecaveye, S.N. Han, K.K. Van de Vijver, F. Amant.

Difficulties with diagnosis of malignancies in pregnancy.

Best Pract Res Clin Obstet Gynaecol (2016) pp. 19-32 
[2] J. de Haan, M. Verheecke, K. Van Calsteren, B. Van Calster, R. G. Shmakov, M. Mhallem Gziri,

Oncological management and obstetric and neonatal outcomes for women diagnosed with

cancer during pregnancy: a 20-year international cohort study of 1170 patients.

Lancet Oncology (2018) pp. 337-346

[3] E. Cardonick.

Cancer occurs in approximately 1 per 1,000 pregnancies.

Oncology (Williston Park) (2008) pp. 22-23

[4] Lee Y.Y., Roberts C.L. , Dobbins T, E. Stavrou , K. Black , J. Morris et al.

Incidence and outcomes of pregnancy-associated cancer in Australia, 1994-2008:

a population-based linkage study.

British Journal Obstetrics Gynaecology (2012) pp. 1572-1582.

[5] L.H. Smith, B. Danielse, M.E. Allen, R. Cress.

Cancer associated with obstetric delivery: results of linkage with the California cancer registry.

American Journal Obstetrics Gynecology (2003) pp. 1128-1135

[6] S. Eibye S, S.K. Kjær, L. Mellemkjær L.

Incidence of pregnancy-associated cancer in Denmark, 1977-2006.

Obstetrics Gynecology (2013) pp. 608-617

[7] F. Parazzini, M Franchi, A. Tavani, E. Negri, F.A.Peccatori.

Frequency of Pregnancy Related Cancer: A Population Based Linkage Study in Lombardy, Italy.

International Journal Gynecology Cancer (2017) pp. 613-619

[8] AIRTUM Working Group, S. Busco, C. Buzzoni, S. Mallone, A. Trama, M. Castaing.

Italian cancer figures--Report 2015: The burden of rare cancers in Italy.

Epidemiologia \& Prevenzione (2016), pp. 1-120

[9] M. Cavalieri, L. Gitto, C. Guccio

Reimbursement systems and quality of hospital care: an empirical analysis for Italy. 
Health Policy (2013) pp. 273-289.

[10] T.M. Andersson, A.L. Johansson, I. Fredriksson, M. Lambe.

Cancer during pregnancy and the postpartum period: a population-based study.

Cancer (2015) pp. 2072-2077 